\title{
Methanolic extract of Physalis minima reduce postmenopausal syndrome in ovariectomized rats
}

\author{
Nur Permatasari, Dian Nugrahenny, Setyawati Soeharto \\ Universitas Brawijaya Indonesia, Indonesia
}

Physalis minima L (P.minima) is an important genus of the Solanaceae family, which contained phyroestrogen withanolides.

The purpose of this study was to explore the effects of methanolic extract P.minima leaf (MEP) on reducing menopause syndromes in ovariectomized rats (OVX).

Twenty female mature Wistar rats were divided into 5 groups: control, OVX control and OVX giving MEP at a dose of 500,1500 and $2500 \mathrm{mg} / \mathrm{kg}$ body weight, respectively for four weeks. Treatment was administered at five weeks after ovariectomy. Body and uterine weight, systolic blood pressure, serum estradiol, lipid profile, isolated aorta response to methacholine, tear volume, the depression-like behavior of ovx rats were studied. Furthermore, we also determined the concentration of withanolide in MEP.

The results showed that OVX had a reduced serum estradiol, uterine weight, and tear volume significantly $(\mathrm{p}<0.05)$ compared with normal. Systolic blood pressure and depressive-like behavior of ovx were significantly increased $(\mathrm{p}<0.05)$. It also showed that ovariectomy impaired the endothelial response to methacholine. Serum total cholesterol, LDL, and HDL of OVX were no significant differences compared with normal. MEP treatment could restore blood pressure, tear volume, serum lipid and depressive-like behavior to the normal level. MEP also decreased the impairment of endothelial response. However, serum estradiol and uterine weight were not different compared with the ovx group. All of the improvement of postmenopausal symptoms have a significant correlation with withanolide concentration contained in MEP.

In conclusion, the decrease of postmenopausal syndrome in ovx rats treated with MEP is probably the role of withanolide that acts as phytoestrogens 